\title{
Avaliação de um programa interlaboratorial de controle de qualidade de resultados de análise de solo ${ }^{1}$
}

\author{
Gustavo Griebeler ${ }^{2}$, Leandro Souza da Silva ${ }^{3}$, Alberto Cargnelutti Filho ${ }^{4}$, Lenio da Silva Santos ${ }^{5}$
}

10.1590/0034-737X201663030014

\section{RESUMO}

O monitoramento da qualidade de análises de amostras de solo contribui para que sejam minimizados os erros nas recomendações de adubação e calagem. Pelo controle de qualidade dos laboratórios da rede ROLAS-RS/SC é avaliada a exatidão média anual dos resultados de quatro amostras, analisadas mensalmente, no ano. Os dados amostrais devem ajustar-se à distribuição normal e não apresentar outliers, para que a mediana possa ser assumida como medida de tendência central. O objetivo deste trabalho foi verificar se os dados de controle de qualidade ajustam-se à distribuição normal, identificar a presença de outliers e como o método de cálculo dos dados pode afetar a estimativa de exatidão dos laboratórios. O teste de Lilliefors identificou a normalidade e os outliers foram identificados pelo método dos quartis. Os outliers foram eliminados para ajustar os dados à distribuição normal. Também foi testado o uso da média em substituição à mediana e avaliado o procedimento de cálculo da exatidão por atributo de análise, em vez da exatidão média de todos os atributos. Somente $48 \%$ dos dados apresentaram distribuição normal. A exclusão dos outliers aumentou de 48 para $65 \%$ o número de análises com distribuição normal, fato que tornou o método de avaliação mais rigoroso. Em dados com distribuição normal, a média parece estimar melhor do que a mediana o valor verdadeiro e o procedimento que calcula a exatidão da média anual pode ocultar atributos com exatidão inferior ao mínimo preconizado pelo sistema.

Palavras-chave: normalidade, outliers, precisão, laboratórios, rede ROLAS-RS/SC.

\section{ABSTRACT}

\section{Evaluation of an interlaboratory quality control program of soil analysis results}

The monitoring of the quality of soil analysis reduces errors in the recommendations of liming and fertilizers. The program of quality control of ROLAS-RS/SC network evaluates the analysis accuracy by testing four soil samples monthly for a year. However, the data sets must have normal distribution and absence of outliers to insure that the median can be considered as an estimation of the true value from the soil samples. Therefore, the objectives of this work were to check the normal distribution, identify outliers and evaluate procedures of accuracy calculations, such as how these aspects may affect laboratory accuracy. The Lilliefors test was run to check the normality and the outliers were identified through the quartile test. The outliers were eliminated from data population. The substitution of the median by the average as criterion of central reference was tested as well as the procedure for calculating the accuracy per attribute instead of annual average accuracy. Only $48 \%$ of the data followed normal distribution. The exclusion of outliers improved the analyzes with normal distribution up to $65 \%$, which decreased laboratories with minimal accuracy required by the network. When data sets have normal distribution, the average shows a better estimation than the median and the procedure of calculating the annual average accuracy may hide attributes with inferior accuracy than the minimal average required.

Key words: normality; outliers; accuracy; laboratories; ROLAS-RS/SC network.

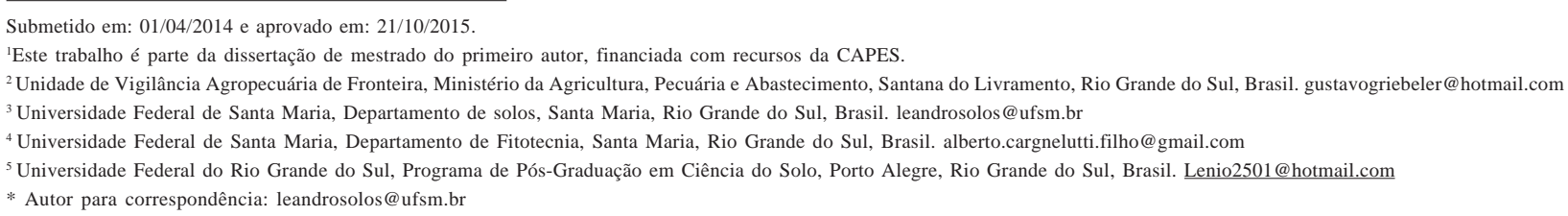




\section{INTRODUÇÃO}

A análise química é o procedimento mais difundido para a avaliação da fertilidade do solo (Bissani et al., 2008) e é de fundamental importância para os sistemas de produção agrícola. A avaliação compreende a coleta do solo, o preparo das amostras, a extração e a determinação dos teores de elementos químicos $\left(\mathrm{P}, \mathrm{K}^{+}, \mathrm{Ca}^{2+}, \mathrm{Mg}^{2+}, \mathrm{H}^{+}, \mathrm{Al}^{3+}\right.$ e índice $\mathrm{SMP}$ ) e de frações do solo de interesse (argila e matéria orgânica), de acordo com métodos preconizados, e a interpretação dos resultados (CQFS, 2004), os quais são denominados neste trabalho como "atributos de análise". A partir dos laudos de análise emitidos pelos laboratórios, os resultados são interpretados de acordo com tabelas, estabelecidas com base em experimentos de calibração, para converter os valores analíticos em informações sobre as necessidades de calagem e de adubação (Schlindwein \& Gianello, 2008). Contudo, para que a análise de solo proporcione a correta recomendação de calagem e adubação, é indispensável que os laboratórios produzam resultados que reflitam com exatidão a disponibilidade de nutrientes do solo. Além disso, análises químicas, quando erradas ou não suficientemente confiáveis, podem representar desperdícios de tempo e de dinheiro (Bode, 1995).

Dessa forma, visando inicialmente a uniformizar os métodos de análise de solos e, posteriormente, controlar a qualidade das análises, em 1968 foi fundada, no sul do Brasil, a Rede Oficial de Laboratórios de Análise de Solo e de Tecidos Vegetais dos Estados do Rio Grande do Sul e de Santa Catarina (ROLAS-RS/SC) (Wiethölter, 2008). Assim, surgiu o programa de controle de qualidade da ROLAS-RS/ $\mathrm{SC}$, que tem por objetivo avaliar os laboratórios por meio da exatidão média anual de quatro amostras mensais. A estimativa da exatidão é determinada a partir da precisão, estabelecida por meio de faixas de distância do desvio padrão da mediana, sendo atribuídos asteriscos (como uma forma de identificação e de contagem) na medida em que as faixas são ultrapassadas. Em outras palavras, cada atributo analisado produz uma população de dados $n$ que corresponde ao número de laboratórios participantes do programa. Obtêm-se a mediana e o desvio padrão, para comparar o resultado do laboratório com o valor mediano (precisão da análise). Conforme os valores se afastem do desvio padrão são-lhes atribuídos asteriscos. Por fim, os asteriscos são somados para compor um índice de exatidão do laboratório. A partir da exatidão, os laboratórios são classificados em conceitos A, B, C e D, sendo que aqueles que obtêm os conceitos A e B recebem o selo de qualidade da ROLAS-RS/SC, para o ano seguinte, e os laboratórios com conceitos C e D permanecem filiados à ROLAS; porém, não recebem o selo e, caso permaneçam nessa condição por três anos consecutivos, são descredenciados (Wiethölter e Dahmer, comunicação pessoal).
No controle de qualidade são utilizadas amostras de solo com concentrações desconhecidas dos atributos analisados e assume-se a mediana dos resultados como o referencial das amostras, pois esta é uma medida de posição definida pelo número central de uma série de dados (Crespo, 2009) e não sofre influência de valores discrepantes ou anômalos (outliers). De acordo com Andrade \& Ogliari (2007), a análise de uma amostra, quando repetida várias vezes, reproduz uma curva simétrica com resultados acima e abaixo do valor da média, a que se denomina curva da distribuição normal ou distribuição de Gauss, de modo que $68,26 \%$ dos dados se afastam em até um desvio padrão da média. Dessa forma, quando se utilizam amostras de solo de que não são conhecidas as concentrações dos elementos (amostras não certificadas por uma instituição de padronização) e toma-se por base a mediana (em vez da média) e o desvio padrão da mediana como referências de precisão, a existência de distribuição normal no conjunto de resultados torna-se condição básica, a ser atendida, para se utilizar a mediana como valor central (estimativa da concentração verdadeira dos elementos da amostra). Quando um conjunto de dados não apresenta distribuição normal, a mediana torna-se uma estivativa pouco confiável. Além disso, a existência de outliers altera a amplitude do desvio padrão, o que afeta o rigor do critério de exatidão, penalizando ou favorecendo alguns laboratórios, de acordo com os resultados obtidos em cada amostra. Portanto, caso não haja distribuição normal dos resultados das análises e,ou, a presença de um grande número de outliers, a análise estatística atualmente adotada pelo controle de qualidade da ROLAS-RS/SC pode não estar avaliando os laboratórios adequadamente.

Outra limitação do sistema pode advir do fato de a classificação conceitual ser baseada na exatidão, uma vez que esta é dependente da média anual de todos os atributos quantificados na análise de solo. Nesse contexto, a existência de uma análise com precisão aquém da adequada pode estar sendo ocultada, por causa do efeito de diluição exercido pelas análises que apresentam alta precisão. Com o sistema atual da ROLAS-RS/SC, o limite inferior para se obter o selo de qualidade (conceito B, que equivale a $85 \%$ de exatidão) admite que 192 determinações entre 432 (quantidade de análises anuais realizadas por cada laboratório dentro do controle de qualidade) estejam abaixo da precisão mínima preconizada. Esta forma de cálculo mascara o fato de estas análises estarem distribuídas entre todos os atributos da análise, ou concentradas em um único deles. Assim, este método possibilita que os laboratórios obtenham conceito A ou B, mesmo existindo algum atributo completamente fora do padrão preconizado.

Além da ROLAS, existem outros programas interlaboratoriais no Brasil: o Programa de Controle de Qualidade 
de Laboratórios com o Sistema IAC de Análise de Solo; o PAQLF (Programa de Análise de Qualidade dos Laboratórios de Fertilidade) - Método da Embrapa Solos; o Programa Interlaboratorial de Controle de Qualidade de Análise de Fertilidade de Solo - Profert e a Comissão Estadual de Laboratórios de Análises Agronômicas do Paraná C.E.L.A. (IAC, 2006). O programa do IAC utiliza o método descrito por Quaggio (1987) e Quaggio et al. (1994), que retira os outliers dos conjuntos de dados e estes recebem um asterisco na medida em que se recalcula o coeficiente de variação, até que este alcance $20 \%$. Os demais programas citados têm um sistema estatístico semelhante ao utilizado pelo IAC e acredita-se que o sistema de controle de qualidade da ROLAS-RS/SC poderia ser aprimorado com alguns ajustes, como a verificação da distribuição normal e a eliminação dos outliers das amostras, a adoção de amostras certificadas (concentrações conhecidas), em substituição à mediana, e a concessão de selos de qualidade somente àqueles que apresentam todos os atributos de análise com exatidão mínima de $85 \%$.

Portanto, o objetivo deste trabalho foi verificar, a partir do banco de dados de resultados de análise de solo do Controle de Qualidade da ROLAS-RS/SC, a normalidade dos dados, a existência de outliers e como estes fatores podem afetar o rigor da classificação conceitual dos laboratórios pelo sistema de Controle de Qualidade, propondo alternativas para as eventuais limitações encontradas.

\section{MATERIAL E MÉTODOS}

Neste trabalho, foram considerados os seguintes termos: a) atributos de análise ou atributos analisados: os elementos químicos, a argila e a matéria orgânica analisados nas amostra de solo; b) determinações: o resultado oriundo da execução de um procedimento analítico; c) precisão: o grau de afastamento do resultado em relação à mediana; d) exatidão: a média obtida a partir da precisão; e) mediana: valor referente ao número localizado na posição central da população de dados, obtido das determinações dos laboratórios de uma amostra de solo.

O sistema de controle de qualidade da ROLAS-RS/SC foi avaliado a partir do banco de dados, do ano de 2009, dos 25 laboratórios que integravam a rede (os laboratórios foram identificados com o mesmo código aplicado pela ROLAS-RS/SC no ano de 2009). O banco de dados foi composto pelos resultados dos atributos de análise $(\mathrm{pH}$ em água, índice SMP, teores de argila e de matéria orgânica, $\mathrm{P}, \mathrm{K}^{+}, \mathrm{Ca}^{2+}, \mathrm{Mg}^{2+}$ e $\mathrm{Al}^{3+}$ ), de 48 amostras (quatro por mês, durante 12 meses), realizadas pelos 25 laboratórios, que totalizaram 10.800 determinações. Os dados foram submetidos a cinco procedimentos de avaliação:

a) O Procedimento $\left(\mathrm{P}_{1}\right)$ compreendeu a execução dos critérios atuais da ROLAS-RS/SC, sendo este o trata- mento referência para a classificação dos laboratórios. Nesse procedimento, é adotada a mediana como a estimativa do valor correto da amostra. Como critério de precisão é utilizada a distância da determinação de um laboratório, em desvio padrão (s) da mediana, em relação à mediana formada pelos resultados dos 25 laboratórios. A precisão do resultado de cada laboratório é identificada por asteriscos, de acordo com os afastamentos, em desvios padrão populacionais $(\mathrm{s}): \leq 1,0 \mathrm{~s} ;>1,0 \mathrm{e} \leq 1,5 \mathrm{~s} ;>1,5 \mathrm{e} \leq 2,0 \mathrm{~s} ; \mathrm{e}>2,0 \mathrm{~s}$ da mediana, para os quais são atribuídos zero, um, dois e três asteriscos, aos resultados que se enquadram dentro desses limites, respectivamente. O método também estabelece algumas exceções para a não aplicação dos asteriscos (Tabela 1), como forma de não penalizar excessivamente os laboratórios, nas situações em que o desvio padrão é muito baixo. A partir do somatório de asteriscos das amostras mensais recebidos por todos os atributos analisados, compõese a exatidão percentual, pela equação elaborada pela ROLAS-RS/SC:

Exatidão $(\%)=100-\left\{\sum_{\text {asteriscos }}\left[\frac{100}{3 \times \mathrm{x}^{\circ} \text { atributos }}\right]\right\}$

sendo que o $n^{o}$ de atributos analisados em 2009 foi de nove.

Com a exatidão percentual é obtido o conceito do laboratório, de acordo com a expressão:

Conceito $=\underline{\text { Exatidão }(\%)}$

48

Tabela 1: Critérios de exceção para cancelamento dos asteriscos no sistema de Controle de Qualidade das análises de solos, em laboratórios pertencentes a ROLAS-RS/SC

\begin{tabular}{|c|c|c|c|}
\hline \multicolumn{2}{|c|}{ Atributos analisados } & \multirow{2}{*}{$\begin{array}{c}\text { Mediana } \\
-\end{array}$} & \multirow{2}{*}{$\begin{array}{c}\begin{array}{c}\text { Valor- } \\
\text { Mediana }^{(1)}\end{array} \\
\leq 0,1\end{array}$} \\
\hline pH e SMP & - & & \\
\hline Argila & $\mathrm{g} \mathrm{dm}^{-3}$ & $\begin{array}{c}\leq 200 \\
>200 \leq 450 \\
>450\end{array}$ & $\begin{array}{l}\leq 10 \\
\leq 20 \\
\leq 30\end{array}$ \\
\hline Matéria orgânica & $\mathrm{g} \mathrm{dm}^{-3}$ & $\leq 1$ & $\leq 1$ \\
\hline $\mathrm{P}$ & $\mathrm{mg} \mathrm{dm}{ }^{-3}$ & $\begin{array}{c}\leq 10 \\
10 \mathrm{~d} " 20 \\
>20\end{array}$ & $\begin{array}{l} \\
\leq 1 \\
\leq 1,25 \\
\leq 1,5\end{array}$ \\
\hline $\mathrm{K}^{+}$ & $\mathrm{mg} \mathrm{dm} \mathrm{m}^{-3}$ & $\begin{array}{c}\leq 50 \\
50 \leq 100 \\
>100\end{array}$ & $\begin{array}{l}\leq 1 \\
\leq 2 \\
\leq 3\end{array}$ \\
\hline $\mathrm{Al}^{3+}$ & $\mathrm{mmol}_{\mathrm{c}} \mathrm{dm}^{-3}$ & $\begin{array}{l}\leq 3 \\
>3\end{array}$ & $\begin{array}{l}\leq 3 \\
\leq 1\end{array}$ \\
\hline $\mathrm{Ca}^{2+}$ & $\mathrm{mmol}_{\mathrm{c}} \mathrm{dm}^{-3}$ & $\begin{array}{l}\leq 100 \\
>100\end{array}$ & $\begin{array}{l}\leq 1 \\
\leq 2\end{array}$ \\
\hline $\mathrm{Mg}^{2+}$ & $\mathrm{mmol}_{\mathrm{c}} \mathrm{dm}^{-3}$ & $\leq 100$ & $\leq 1$ \\
\hline
\end{tabular}

${ }^{(1)}$ Em módulo. 
O denominador da expressão acima é originado da multiplicação das quatro amostras mensais pelo número de meses do ano. Os laboratórios recebem os conceitos em função dessa exatidão média, sendo que a exatidão $\geq$ $90 \%$ confere conceito A, enquanto os laboratórios com exatidão $<90 \%$ e $\geq 85 \%$; $<85 \%$ e $\geq 80 \%$ e $<80 \%$ recebem conceitos B, C e D, respectivamente (Wiethölter \& Dahmer, comunicação pessoal).

b) O Procedimento $\left(\mathrm{P}_{2}\right)$ envolveu a aplicação dos critérios da ROLAS-RS/SC (mesmos critérios do $\mathrm{P}_{1}$ ), incluindo o teste de distribuição normal antes da aplicação dos critérios e, no caso de constatada a não normalidade, a aplicação do teste dos quartis (Andrade \& Ogliari, 2007) para identificar a presença de outliers, sendo estes eliminados do conjunto de dados. Ao serem eliminados, os outliers automaticamente receberam três asteriscos e para o restante dos dados da análise foi recalculada a mediana e o desvio padrão da mediana, sendo novamente submetidos aos critérios descritos no $\mathrm{P}_{1}$. Para verificar a distribuição normal, foi aplicado o teste de Lilliefors (Campos, 1983), utilizando-se o software Bioestat 5.0, e o teste dos quartis, utilizando-se o Microsoft Office Excel 2007, para identificar os outliers e eliminá-los. O critério de eliminação de outliers foi estabelecido, segundo descrito por Andrade \& Ogliari (2007), que classificam como outliers aqueles valores uma vez e meia inferiores ao primeiro quartil $\left(\mathrm{Q}_{1}-1,5 D I\right)$, ou uma vez e meia superiores ao terceiro quartil $\left(\mathrm{Q}_{3}+\right.$ $1,5 D I)$, sendo $\mathrm{Q}_{1}$ o primeiro quartil, $\mathrm{Q}_{3}$ o terceiro quartil e DI a distância interquartílica $\left(D I=\mathrm{Q}_{3}-\mathrm{Q}_{1}\right)$. Valores além desses limites, dentro de uma distribuição normal, constituem $0,7 \%$ dos resultados (Andrade \& Ogliari, 2007). Nesse procedimento, as amostras sem distribuição normal e sem possibilidade de ajuste pela eliminação de outliers permaneceram no banco de dados para compor a exatidão média.

c) O Procedimento $\left(\mathrm{P}_{3}\right)$ seguiu a mesma metodologia descrita em $\mathrm{P}_{2}$, porém as amostras sem distribuição normal, que incluíram todas as análises de $\mathrm{pH}$ e índice SMP, foram descartadas do banco de dados, portanto, não compuseram a exatidão média.

d) O Procedimento $\left(\mathrm{P}_{4}\right)$ analisou a exatidão somente das amostras com distribuição normal pelos critérios descritos em $\mathrm{P}_{1}$, porém, utilizando a média e o desvio padrão da média em substituição à mediana e ao desvio padrão da mediana.

e) O Procedimento $\left(\mathrm{P}_{5}\right)$ estabeleceu um novo critério para conceituar os laboratórios. Os critérios para o recebimento de asteriscos foram mantidos de acordo com $\mathrm{P}_{1}$, porém, a exatidão média anual foi calculada para cada atributo analisado, pela modificação da equação 1 :
Exatidão $(\%)=100-\left\{\left[\frac{\sum_{\text {asteriscos do atributo }}}{48}\right] \times\left[\frac{100}{3 \times n^{\circ} \text { atributos }}\right]\right\}$

sendo utilizado 1 ao $n^{o}$ de atributos, pois os atributos foram avaliados individualmente.

A partir da exatidão por atributo, foi estabelecido um critério numérico que transforma os conceitos obtidos em pontos, de modo que para obter conceito A, o laboratório não pode apresentar nenhuma análise com conceito inferior a A. Para tanto, o conceito A recebeu um valor arbitrário igual a 1 e os demais foram calculados pela equação:

$\left(n^{\underline{o}}\right.$ atributos -1$)+Y_{\mathrm{i}-1}>\left(n^{\underline{o}}\right.$ atributos $\left.Y_{\mathrm{i}-1}\right)+1$

sendo o $n^{o}$ de atributos o número de atributos analisados (9), $Y$ as faixas de exatidão que originam os conceitos e $i$ a ordem das faixas, considerando-se A, B, C e D como $1^{\mathrm{a}}, 2^{\mathrm{a}}$, $3^{\mathrm{a}}$ e $4^{\mathrm{a}}$ faixas, respectivamente. Assim, atribuindo-se 1 para o conceito A, foram obtidos os valores 2,11 e 92 para os conceitos B, C e D, respectivamente. Os somatórios máximos de pontos para obtenção dos conceitos A, B e C foram 9, 18 e 99, respectivamente. Dessa forma, para se obter conceito A, o somatório das nove amostras não pode exceder a nove pontos. Caso uma amostra contenha oito conceitos A e apenas um conceito B, o somatório passa a ser dez, impedindo a obtenção de conceito A e assim sucessivamente. Porém, mesmo neste procedimento, receberiam Selo de Qualidade os laboratórios que alcançarem a exatidão mínima preconizada pela ROLAS-RS/ SC ( $85 \%$; conceitos A e B).

Por fim, foram comparados quanto ao rigor, os procedimentos $\mathrm{P}_{1}, \mathrm{P}_{2}, \mathrm{P}_{3}$ e $\mathrm{P}_{4}$, entre si, em função da classificação dos laboratórios em conceitos $\mathrm{A}, \mathrm{B}, \mathrm{C}$ e D, e o $\mathrm{P}_{5}$ avaliou-se a sensibilidade do sistema pela comparação entre a média de todas as análises que compõem a exatidão anual com o sistema de pontos.

\section{RESULTADOS E DISCUSSÃO}

Dentre os 25 laboratórios, integrantes da rede no ano de 2009, pelo procedimento $\mathrm{P}_{1}$ foram conceituados com A, B, C e D, 12, 7, 4 e 2 laboratórios, respectivamente (Tabela 2). Com esta distribuição, o sistema concederia selos de qualidade para 19 laboratórios $(12 \mathrm{~A}+7 \mathrm{~B})$. Ao aplicar o procedimento $\mathrm{P}_{2}$, foi observado que a média das determinações com distribuição normal foi de $48 \%$ (Tabela 3). Dessa forma, pode-se inferir que os pressupostos estatísticos que sustentam o uso da mediana como valor referencial não foram atendidos em $52 \%$ das determinações do ano de 2009. Observou-se ausência de distribuição normal em grande número de determinações de $\mathrm{pH}$ e de índice SMP, as quais são expressas em base logarítmica, que concentra os valores próximos à mediana e, portanto, não seguem distribuição normal. Assim, a eliminação de outliers não se justifica para esses atributos. Em relação 
ao controle de qualidade, o sistema da ROLAS-RS/SC prevê algumas regras de cancelamento de aplicação dos asteriscos (Tabela 1), para não penalizar os resultados numericamente próximos à mediana e com faixas de intervalos estreitos de desvios padrão. Porém, esses limites foram estabelecidos arbitrariamente no sistema, sendo que, dentro deles ainda poderia haver algum tipo de influência da não normalidade dos dados.

Ao eliminar os outliers pelo procedimento $\mathrm{P}_{2}$, os casos com distribuição normal aumentaram para 65\% (Tabela 3). Embora o $\mathrm{P}_{2}$ tenha corrigido apenas uma parcela dos dados, houve aumento do rigor do controle, indicado pela diminuição do número de conceitos A (Tabela 2). Além de pH e de índice SMP, anteriormente citados, foi observado que uma fração alta das determinações, principalmente de

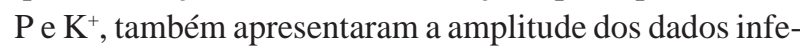
rior a 1,5 DI, o que impossibilitou ajustar todos os dados à distribuição normal. Em princípio, essa característica indicaria que os laboratórios seriam precisos na determinação desses atributos, se os valores obtidos estivessem dentro dos limites de detecção dos equipamentos analíticos (faixa de trabalho), considerados na avaliação, como ocorre com pH e SMP. Entretanto, nas determinações de $\mathrm{P}$ são frequentes os resultados de amostras com valores inferiores a 3,0 $\mathrm{mg} \mathrm{dm}^{-3}$, superiores a $50 \mathrm{mg} \mathrm{dm}^{-3}$, e, no caso do $\mathrm{K}^{+}$, superiores a $150 \mathrm{mg} \mathrm{dm}^{-3}$. Nestes casos, os resultados que extrapolam a faixa de trabalho passam a ser considerados pelo Controle de Qualidade como 3,0, 50 e $150 \mathrm{mg} \mathrm{dm}^{-3}$, respectivamente, de acordo com a faixa de trabalho (Tabela 4) utilizada pelos laboratórios da ROLAS-RS/SC. Dessa forma, tomando-se o P como exemplo, quando a concentração do elemento na amostra de solo excede os limites inferior ou superior determinado, o resultado de todos os laboratórios é 3,0 $\mathrm{mg} \mathrm{dm}^{-3}$ ou $50 \mathrm{mg}$ $\mathrm{dm}^{-3}$, respectivamente. Neste caso, a amplitude corresponde a zero e, a mediana, ao valor limite da faixa de trabalho; portanto, a mediana não estima o valor verdadeiro dessas amostras, que podem ser consideradas inadequadas para discriminar a precisão dos laboratórios e prejudiciais ao cálculo da exatidão.

Para atender à prerrogativa de que, por meio da distribuição normal, pode-se assumir a mediana como estimativa do valor verdadeiro da amostra, a exclusão dos ouliers faz-se necessária e isto acarreta consequências sobre a

Tabela 2: Classificação conceitual dos laboratórios, segundo modificações no sistema atual da ROLAS-RS/SC, com base nos resultados do Controle de Qualidade de 2009

\begin{tabular}{lcccc}
\hline Procedimento* $^{*}$ & \multicolumn{4}{c}{ Conceito dos laboratórios } \\
\cline { 2 - 5 } & $\mathbf{A}$ & $\mathbf{B}$ & $\mathbf{C}$ & $\mathbf{D}$ \\
\hline $\mathrm{P}_{1}$ & 12 & 7 & 4 & 2 \\
$\mathrm{P}_{2}$ & 9 & 10 & 5 & 1 \\
$\mathrm{P}_{3}$ & 7 & 7 & 7 & 4 \\
$\mathrm{P}_{4}$ & 8 & 11 & 5 & 1 \\
$\mathrm{P}_{5}$ & 1 & 4 & 5 & 15 \\
\hline
\end{tabular}

* $\mathrm{P}_{1}$ :atual sistema empregado pela ROLAS. $\mathrm{P}_{2}: \mathrm{P}_{1}$ com dados ajustados à distribuição normal por meio de eliminação de outliers. $\mathrm{P}_{3}$ : $\mathrm{P}_{2}$ utilizando somente as amostras com distribuição normal. $\mathrm{P}_{4}: \mathrm{P}_{3}$ com substituição da mediana por média e desvio padrão da mediana por desvio padrão da média. $\mathrm{P}_{5}$ : cálculo da exatidão por análise e conversão em pontos para a atribuição do conceito.

Tabela 3: Percentual de análises de solo que seguem a distribuição normal nos resultados do Controle de Qualidade da ROLAS-RS/ SC de 2009

\begin{tabular}{|c|c|c|c|c|}
\hline \multicolumn{5}{|c|}{ Atributos que seguem a distribuição normal $(\%)^{(1)}$} \\
\hline & \multicolumn{2}{|c|}{$\mathrm{DN}^{(2)}$} & \multicolumn{2}{|c|}{ DN corrigida } \\
\hline & Sim & Não & Sim & Não \\
\hline$\overline{\mathrm{pH}}$ & 0,5 & 99,5 & - & - \\
\hline SMP & 19 & 81 & - & - \\
\hline $\mathrm{MO}^{(3)}$ & 48 & 52 & 63 & 38 \\
\hline Argila & 73 & 27 & 90 & 10 \\
\hline $\mathrm{Al}^{3+}$ & 52 & 48 & 54 & 46 \\
\hline $\mathrm{Ca}^{2+}$ & 75 & 25 & 92 & 8 \\
\hline $\mathrm{Mg}^{2+}$ & 50 & 50 & 77 & 23 \\
\hline $\mathrm{P}$ & 48 & 52 & 60 & 40 \\
\hline $\mathrm{K}^{+}$ & 17 & 83 & 19 & 81 \\
\hline Média $^{(4)}$ & 48 & 52 & 65 & 35 \\
\hline
\end{tabular}

(1)\% sobre 10.800 amostras. ${ }^{(2)}$ Distribuição normal. ${ }^{(3)}$ Matéria orgânica. ${ }^{(4)}$ Após eliminação dos outliers (com exceção do pH e SMP). 
amplitude do desvio padrão da mediana. Embora a mediana não seja afetada por outliers, o desvio padrão aumenta ou diminui com a existência deles, tornando mais brandos ou rigorosos, respectivamente, os critérios de exatidão empregados. A classificação dos laboratórios foi mais rigorosa eliminando-se os outliers das amostras; no entanto, por causa do total de asteriscos recebidos este efeito não foi perceptível (Tabela 5). A exatidão média anual obtida em cada procedimento (Tabela 2) indica que houve redistribuição dos conceitos, quando aplicado o sistema de ajuste dos dados à distribuição normal. Utilizando-se $\mathrm{o} \mathrm{P}_{{ }_{1},}$ foram concedidos selos de qualidade para $19\left(12^{\mathrm{a}}+\right.$ 7B) dos 25 laboratórios e, ao se ajustar à distribuição normal $\left(\mathrm{P}_{2}\right)$, este número manteve-se, mas diminuíram os laboratórios com conceito $\mathrm{A}\left(9^{\mathrm{a}}+10 \mathrm{~B}\right)$.

Nestes procedimentos, cabe salientar que os outliers receberam três asteriscos, como os demais dados que permaneceram na amostra e assumiram afastamento superior a dois desvios padrão da mediana, depois de recalculadas a mediana e o desvio padrão da mediana. Isto explica as diferenças discretas entre os procedimen- tos $\mathrm{P}_{1}$ e $\mathrm{P}_{2}$. Possivelmente, a adoção de critérios que penalizam com maior rigor os outliers, a exemplo do sistema descrito por Quaggio et al. (1994), utilizado nos laboratórios do Estado de São Paulo, discriminaria com maior rigor os laboratórios e esta lógica poderia ser implementada, no caso de o procedimento $\mathrm{P}_{2}$ ser aplicado na ROLAS-RS/SC.

$O$ procedimento $\mathrm{P}_{3}$ foi ainda mais rigoroso que o $\mathrm{P}_{2}$. Neste, somente 14 laboratórios receberiam selo de qualidade e este efeito pode ser associado ao descarte de $35 \%$ das determinações que não apresentavam distribuição normal. Esta parcela das determinações apresentava a distribuição dos dados de forma semelhante às do $\mathrm{pH}$ e do índice SMP (amplitude < 1,5 DI), indicando que os dados são mais precisos e, por este motivo, não devem ser eliminados do banco de dados. Portanto, $\mathrm{o}_{3}$ não foi adequado para avaliar a exatidão dos laboratórios.

O procedimento $\mathrm{P}_{4}$, que adotou a média como estimativa do valor verdadeiro, foi mais rigoroso que os procedimentos $\mathrm{P}_{1}$ e $\mathrm{P}_{2}$, mas menos rigoroso que $\mathrm{P}_{3}$. Quando os dados seguem a distribuição normal, as

Tabela 4: Limites de uso dos resultados no Controle de Qualidade dos elementos analisados pelos laboratórios da ROLAS-RS/SC em 2009

Limites adotados nos resultados de laboratórios da ROLAS-RS/SC

\begin{tabular}{lccc}
\hline Atributo de análise & Unidade & Limite inferior & Limite superior \\
\hline pH e SMP & - & 4 & 8,5 \\
Matéria orgânica & $\mathrm{g} \mathrm{dm}^{-3}$ & 10 & 70 \\
Argila & $\mathrm{g} \mathrm{dm}^{-3}$ & 100 & 700 \\
$\mathrm{P}$ & $\mathrm{mg} \mathrm{dm}^{-3}$ & 3 & 50 \\
$\mathrm{~K}^{+}$ & $\mathrm{mg} \mathrm{dm}^{-3}$ & 10 & 150 \\
$\mathrm{Al}^{3+}$ & $\mathrm{mmol}_{\mathrm{c}} \mathrm{dm}^{-3}$ & 0 & 70 \\
$\mathrm{Ca}^{2+}$ & $\mathrm{mmol}_{\mathrm{c}} \mathrm{dm}^{-3}$ & 2 & 150 \\
$\mathrm{Mg}^{2+}$ & $\mathrm{mmol}_{\mathrm{c}} \mathrm{dm}^{-3}$ & 2 & 100 \\
\hline
\end{tabular}

Tabela 5: Número de asteriscos recebidos por análise em função do desvio padrão (s) da mediana nos resultados do Controle de Qualidade da ROLAS-RS/SC em 2009, com e sem ajuste da distribuição normal (remoção de outliers)

\begin{tabular}{|c|c|c|c|c|c|c|c|c|c|c|}
\hline \multirow{2}{*}{ Classe } & \multicolumn{10}{|c|}{ Número de asteriscos por classe de desvio padrão (s) da mediana } \\
\hline & pH & SMP & MO $^{(1)}$ & Mg & Al & $\mathbf{P}$ & Argila & $\mathbf{C a}$ & $\mathbf{K}$ & Totais \\
\hline & \multicolumn{10}{|c|}{ Dados não ajustados à distribuição normal } \\
\hline $\mathrm{s} \leq 1\left(\mathrm{sem}^{*}\right)$ & 1104 & 978 & 938 & 946 & 1016 & 992 & 962 & 909 & 957 & 7266 \\
\hline $\mathrm{s}>1 \leq 1,5(*)$ & 5 & 88 & 140 & 128 & 99 & 104 & 120 & 158 & 134 & 976 \\
\hline $\mathrm{s}>1,5 \leq 2,0(* *)$ & 33 & 74 & 54 & 60 & 46 & 50 & 63 & 74 & 54 & 1016 \\
\hline $\mathrm{s}>2,0(* * *)$ & 58 & 60 & 68 & 66 & 39 & 54 & 55 & 483 & 55 & 1542 \\
\hline \multirow[t]{2}{*}{ Total de asteriscos ${ }^{(2)}$} & 245 & 416 & 452 & 446 & 308 & 366 & 411 & 291 & 407 & 3534 \\
\hline & \multicolumn{10}{|c|}{ Dados ajustados à distribuição normal } \\
\hline $\mathrm{s} \leq 1\left(\mathrm{sem}^{*}\right)$ & 1191 & 927 & 860 & 880 & 1001 & 955 & 887 & 852 & 920 & 8473 \\
\hline $\mathrm{s}>1 \leq 1,5(*)$ & 5 & 126 & 167 & 147 & 103 & 115 & 145 & 182 & 149 & 1139 \\
\hline $\mathrm{s}>1,5 \leq 2,0(* *)$ & 1 & 87 & 73 & 67 & 52 & 60 & 86 & 91 & 58 & 575 \\
\hline $\mathrm{s}>2,0(* * *)$ & 3 & 60 & 100 & 106 & 44 & 70 & 82 & 75 & 73 & 613 \\
\hline Total de asteriscos ${ }^{(2)}$ & 16 & 480 & 613 & 599 & 339 & 445 & 563 & 589 & 484 & 3515 \\
\hline
\end{tabular}


medidas de tendência central, como moda, média e mediana são coincidentes (Cargnelutti Filho et al., 2009) e, dessa forma, para aquelas amostras que não apresentavam, e posteriormente foram ajustadas, a distribuição normal, supõe-se que o rigor do sistema, ao usar a mediana ou a média, seja idêntico. Porém, o teste de normalidade de Lilliefors (Campos, 1983) compara a distância entre a curva da distribuição teórica e a curva cumulativa, o que admite um grau de assimetria (Campos, 1983). A média pode ser influenciada pela frequência de valores e deslocar o valor central e a amplitude do desvio padrão, enquanto a mediana mantém-se, na maioria das vezes, estática em relação aos ajustes realizados no banco de dados, o que pode penalizar indevidamente os valores próximos aos limites de afastamento $\left(\mathrm{P}_{2}\right)$. Portanto, parece mais adequado utilizar a média do que a mediana, quando as amostras apresentam distribuição normal, pois isso atende com maior fidelidade aos pressupostos estatísticos.

O número total de conceitos recebidos por classe de exatidão foi alterado, em função do procedimento aplicado. Alguns laboratórios, com conceito A obtido pelo $\mathrm{P}_{1}$ obtiveram conceito $\mathrm{D}$, quando submetidos ao $\mathrm{P}_{2}$. O rigor aumentou para os laboratórios que, por exemplo, não apresentavam determinações de $\mathrm{K}^{+}$com resultados inferiores a $150 \mathrm{mg} \mathrm{dm}^{-3}$, naquelas amostras de solos que ultrapassavam a faixa de trabalho (10 a $150 \mathrm{mg} \mathrm{dm}^{-3}$, Tabela 4). Nesses casos, os laboratórios foram beneficiados pelas amostras com concentração do elemento superior à faixa de trabalho, uma vez que estas não detectam as superestimativas e diluem este efeito ao longo do ano. Desse modo, alguns laboratórios com conceito A passaram ao conceito $\mathrm{D}$, quando descartadas as amostras sem distribuição normal, advindas de amostras com concentrações dos elementos que excediam a faixa de trabalho. Por outro lado, houve situações em que o rigor diminuiu. O conceito D obtido por alguns laboratórios, a partir de amostras sem ajuste à distribuição normal $\left(\mathrm{P}_{1}\right)$, passou para A, quando utilizadas apenas amostras com distribuição normal $\left(\mathrm{P}_{3}\right.$ e $\left.\mathrm{P}_{4}\right)$. Neste caso foi observado que esses laboratórios, frequentemente, apresentavam resultados de $\mathrm{K}$ inferiores a $150 \mathrm{mg} \mathrm{dm}^{-3}$, naquelas amostras com teor superior à faixa de trabalho. Portanto, quando a mediana correspondia a $150 \mathrm{mg} \mathrm{dm}^{-3}$, o laboratório recebia asteriscos nessas amostras. Ao eliminá-las, esse fenômeno não foi mais observado. Contudo, isso não significa que o laboratório estivesse subestimando o valor verdadeiro e nem assegura a exatidão daquelas avaliações, pois os valores inferiores a $150 \mathrm{mg} \mathrm{dm}^{-3}$ podem representar resultados inferiores ao primeiro quartil de uma distribuição normal.

Ao se aplicarem os procedimentos $\mathrm{P}_{2}, \mathrm{P}_{3}$ e $\mathrm{P}_{4}$, foi observado que os conceitos obtidos pelos laboratórios não foram coerentes com o rigor imposto pelo ajuste estatístico de cada procedimento. Dessa forma, pode-se inferir que o uso de uma medida de tendência central não é adequado para ser empregado como uma referência para a calibração interna dos laboratórios para os métodos analíticos. Em função desses resultados, o controle de qualidade poderia ser aprimorado com a adoção de um método que empregasse uma referência por meio de amostras certificadas, de valores conhecidos, em substituição a medidas de tendência central, uma vez que a qualidade de um resultado analítico é verificada por meio da incerteza a ele associada (Chui et al., 2004). Os materiais de referência seriam amostras que apresentassem um ou mais valores de propriedades suficientemente homogêneas e bem estabelecidas, de maneira a poderem ser empregados na calibração de um instrumento de medição, na validação de um método analítico, ou na atribuição de valores a um dado material (INMETRO, 2007).

Assim, poderia ser considerada, dentro dos critérios de exatidão, a variabilidade inerente ao método de análise, associada a um desvio padrão conhecido, sendo os asteriscos atribuídos em função do afastamento desse desvio, de acordo com o método atualmente aplicado. Dessa forma, evitar-se-ia que a variabilidade interlaboratorial (objetivo do controle de qualidade) seja superior à variabilidade do método no laboratório (variabilidade intralaboratorial) e que os atributos das análises básicas que não apresentam distribuição normal, como pH e índice SMP, ou em função da concentração dos dados, estabeleçam penalizações em resultados de laboratórios com pequenos desvios em relação à mediana, sendo que isto pode não apresentar implicações agronômicas importantes e causar descréditos desnecessários dos usuários da rede.

Observa-se que o conceito obtido, por meio da exatidão média dos nove atributos de análises realizadas ao longo do ano $\left(\mathrm{P}_{1}\right)$, não revela aquelas com exatidão insuficiente em alguns atributos (Tabela 7). Ao avaliar os conceitos individuais de cada atributo, estabelecendo valores numéricos com pesos distintos para cada conceito (procedimento $\mathrm{P}_{5}$ ), somente cinco dos 19 laboratórios com selo de qualidade (exatidão média > 85\%) em 2009 não possuíam nenhum atributo com conceito inferior a $\mathrm{B}$ e três laboratórios com conceito A apresentaram pelo menos um atributo com conceito D. Como exemplo, o laboratório 49, que obteve conceito A (94\% de exatidão média anual) pelo $\mathrm{P}_{1}$, apresentou os atributos $\mathrm{P}$ e K com conceitos $\mathrm{C}$ e $\mathrm{D}$, respectivamente, quando avaliados pelo $\mathrm{P}_{5}$ (Tabela 7). Este laboratório deteve a terceira melhor exatidão da ROLAS-RS/SC, de acordo com $\mathrm{P}_{1}$, em 2009, porém, em função de apresentar dois atributos com exatidão insuficientes, a exatidão decaiu para a $12^{\text {a }}$ colocação. Também foi verificado que, dentre os laboratórios que receberam 


\begin{tabular}{|c|c|c|c|c|c|c|c|c|c|c|c|c|c|c|}
\hline \multirow{2}{*}{ Lab. } & \multirow{2}{*}{$\begin{array}{c}\text { Exatidão } \\
(\%)\end{array}$} & \multirow{2}{*}{$\begin{array}{l}\text { Conceito } \\
\text { ROLAS }\end{array}$} & \multicolumn{9}{|c|}{ Conceito por atributo } & \multirow{2}{*}{ Pont. ${ }^{(1)}$} & \multirow{2}{*}{$\begin{array}{c}\text { Novo } \\
\text { Conc. }{ }^{(2)}\end{array}$} & \multirow{2}{*}{ Coloc. $^{(3)}$} \\
\hline & & & Argila & pH & SMP & $\mathbf{P}$ & $\mathbf{K}^{+}$ & MO & $\mathbf{A l}^{2+}$ & $\mathrm{Ca}^{2+}$ & $\mathrm{Mg}^{2+}$ & & & \\
\hline 41 & 96 & A & $\mathrm{A}$ & A & A & $\mathrm{A}$ & A & $\mathrm{A}$ & A & A & $\mathrm{A}$ & 9 & $\mathrm{~A}$ & $1^{\circ}$ \\
\hline 01 & 94 & $\mathrm{~A}$ & B & A & $\mathrm{A}$ & $\mathrm{A}$ & A & $\mathrm{A}$ & $\mathrm{B}$ & $\mathrm{A}$ & $\mathrm{A}$ & 11 & $\mathrm{~B}$ & $4^{\circ}$ \\
\hline 49 & 94 & $\mathrm{~A}$ & A & A & A & $\mathrm{C}$ & $\mathrm{D}$ & $\mathrm{A}$ & $\mathrm{A}$ & A & $\mathrm{A}$ & 110 & $\mathrm{D}$ & $12^{\circ}$ \\
\hline 73 & 94 & A & $\mathrm{B}$ & A & A & A & A & A & A & $\mathrm{B}$ & A & 11 & $\mathrm{~B}$ & $5^{\circ}$ \\
\hline 37 & 93 & $\mathrm{~A}$ & A & A & A & $\mathrm{A}$ & A & A & A & A & B & 10 & B & $2^{o}$ \\
\hline 98 & 93 & A & A & A & A & B & A & A & A & A & $\mathrm{A}$ & 10 & B & $3^{\circ}$ \\
\hline 96 & 93 & A & A & A & $\mathrm{C}$ & B & A & $\mathrm{A}$ & $\mathrm{A}$ & A & A & 20 & $\mathrm{C}$ & $7^{\circ}$ \\
\hline 132 & 92 & A & A & A & A & A & $\mathrm{C}$ & A & A & B & A & 20 & $\mathrm{C}$ & $6^{\circ}$ \\
\hline 86 & 91 & A & B & A & $\mathrm{C}$ & A & A & $\mathrm{C}$ & A & A & B & 31 & $\mathrm{C}$ & $8^{\circ}$ \\
\hline 135 & 91 & $\mathrm{~A}$ & A & A & A & $\mathrm{C}$ & A & B & $\mathrm{D}$ & $\mathrm{C}$ & $\mathrm{A}$ & 121 & $\mathrm{D}$ & $14^{\circ}$ \\
\hline 18 & 91 & A & A & A & B & $\mathrm{C}$ & A & $\mathrm{D}$ & A & A & A & 111 & $\mathrm{D}$ & $13^{\circ}$ \\
\hline 88 & 90 & A & A & A & $\mathrm{C}$ & A & A & A & $\mathrm{C}$ & $\mathrm{C}$ & A & 39 & $\mathrm{C}$ & $9^{\circ}$ \\
\hline 54 & 89 & B & A & A & B & A & A & A & B & B & $\mathrm{D}$ & 103 & $\mathrm{D}$ & $11^{\circ}$ \\
\hline 116 & 89 & B & A & A & A & A & A & $\mathrm{C}$ & $\mathrm{C}$ & B & $\mathrm{C}$ & 40 & $\mathrm{C}$ & $10^{\circ}$ \\
\hline 17 & 88 & B & A & A & A & A & A & $\mathrm{D}$ & A & $\mathrm{D}$ & $\mathrm{D}$ & 282 & $\mathrm{D}$ & $21^{\circ}$ \\
\hline 61 & 88 & B & A & A & A & B & A & $\mathrm{D}$ & A & B & $\mathrm{D}$ & 193 & $\mathrm{D}$ & $17^{\circ}$ \\
\hline 83 & 88 & B & B & A & A & B & A & B & $\mathrm{A}$ & $\mathrm{D}$ & $\mathrm{D}$ & 194 & $\mathrm{D}$ & $18^{\circ}$ \\
\hline 153 & 88 & B & $\mathrm{C}$ & A & B & A & $\mathrm{C}$ & A & A & $\mathrm{D}$ & $\mathrm{C}$ & 131 & $\mathrm{D}$ & $15^{\circ}$ \\
\hline 84 & 86 & B & B & A & $\mathrm{D}$ & A & $\mathrm{D}$ & A & $\mathrm{A}$ & $\mathrm{C}$ & B & 203 & $\mathrm{D}$ & $19^{\circ}$ \\
\hline 72 & 84 & $\mathrm{C}$ & A & B & B & $\mathrm{D}$ & $\mathrm{C}$ & $\mathrm{C}$ & $\mathrm{A}$ & $\mathrm{C}$ & $\mathrm{D}$ & 223 & $\mathrm{D}$ & $20^{\circ}$ \\
\hline 147 & 84 & $\mathrm{C}$ & $\mathrm{D}$ & B & B & B & B & $\mathrm{C}$ & $\mathrm{C}$ & A & $\mathrm{C}$ & 134 & $\mathrm{D}$ & $16^{\circ}$ \\
\hline 29 & 84 & $\mathrm{C}$ & $\mathrm{C}$ & A & A & $\mathrm{D}$ & A & $\mathrm{D}$ & $\mathrm{A}$ & $\mathrm{D}$ & $\mathrm{A}$ & 292 & $\mathrm{D}$ & $23^{\circ}$ \\
\hline 141 & 84 & $\mathrm{C}$ & B & A & $\mathrm{D}$ & A & $\mathrm{D}$ & B & B & $\mathrm{D}$ & B & 286 & $\mathrm{D}$ & $22^{\circ}$ \\
\hline 25 & 79 & $\mathrm{D}$ & $\mathrm{D}$ & $\mathrm{D}$ & $\mathrm{D}$ & A & $\mathrm{D}$ & A & $\mathrm{A}$ & B & $\mathrm{C}$ & 384 & $\mathrm{D}$ & $24^{\circ}$ \\
\hline 09 & 71 & $\mathrm{D}$ & $\mathrm{D}$ & A & $\mathrm{D}$ & $\mathrm{D}$ & $\mathrm{C}$ & $\mathrm{D}$ & $\mathrm{A}$ & $\mathrm{D}$ & $\mathrm{D}$ & 565 & $\mathrm{D}$ & $25^{\circ}$ \\
\hline
\end{tabular}

(1)Para compor a pontuação, os conceitos A, B, C e D dos nove atributos de análise foram substituídos pelos valores $1,2,11$ e 92 , respectivamente, e somados. ${ }^{(2)}$ Os limites superiores de cada conceito são: 9, 18 e 99 para A, B e C, respectivamente, a partir da pontuação obtida. ${ }^{(3)}$ Ordenação de acordo com a pontuação. 
conceito B, em 2009, há pelo menos um atributo com conceito $\mathrm{D}$, além de outras combinações de conceitos $\mathrm{C}$ e, ou, D. Portanto, além de adotar o valor de amostras certificadas como referência, em substituição à mediana, o sistema de controle de qualidade da ROLAS-RS/SC pode ser aperfeiçoado pelo estabelecimento da exatidão mínima de $85 \%$ para cada atributo analisado. Deste modo, seria reduzida a possibilidade de causar prejuízos, por inexatidão dos resultados das análises emitidas nos laudos para a recomendação da calagem e adubação, objetivo final da análise de solo.

Entretanto, a mudança dos critérios para avaliação da qualidade das análises de solos realizadas por laboratórios deve ser avaliada com cautela e de forma que seja garantida a qualidade analítica, por meio de critérios estatísticos associados à relevância agronômica. Dessa forma, não se comprometeria de maneira desnecessária a credibilidade dos laboratórios, em função de critérios puramente estatísticos e rigorosos, e seria aprimorada a qualidade dos laudos de análise e, consequentemente, as recomendações de adubação e calagem.

\section{CONCLUSÕES}

As determinações das amostras de solos que originam conjuntos de dados sem distribuição normal pela presença de outliers distorcem a exatidão, sendo pertinente considerar a possibilidade de penalizar os outliers com rigor diferenciado, em eventuais modificações dos critérios do Controle de Qualidade da ROLAS-RS/SC.

As determinações das amostras que originam conjuntos de dados sem distribuição normal, por ultrapassarem as faixas de trabalho, devem ser eliminadas do banco de dados, enquanto aquelas enquadradas dentro das faixas de trabalho e que apresentam amplitude $<1,5$ DI não devem ser excluídas para o cálculo da exatidão.

Em conjuntos de dados com distribuição normal, a média atende melhor aos pressupostos estatísticos do que a mediana, estimando mais adequadamente o valor verdadeiro das amostras. Entretanto, utilizar amostras de solo certificadas possivelmente seja mais apropriado para a calibração interna dos métodos analíticos nos laboratórios.

O procedimento de cálculo da exatidão média anual não revela os atributos que eventualmente não alcançam a exatidão mínima de $85 \%$.

\section{AGRADECIMENTOS}

Agradecemos ao Dr. Sírio Wiethölter, da EMBRAPA Trigo, e à ROLAS-RS/SC, pela cessão dos dados do Controle de Qualidade, para a realização do trabalho, e à CAPES e ao CNPq, pelos auxílios financeiros e pela bolsa de estudos e de Produtividade em Pesquisa.

\section{REFERÊNCIAS}

Andrade DF \& Ogliari PJ (2007) Estatística para as ciências agrárias e biológicas com noções de experimentação. $1^{\mathrm{a}}$ ed. Florianópolis, UFSC. 432p.

Bissani CA, Gianello C, Camargo FAO \& Tedesco MJ (2008) Fertilidade dos solos e manejo da adubação das culturas. Porto Alegre, Metrópole. 344p.

Bode P (1995) Quality management and laboratory accreditation at a university: what can be learned from experience? Analyst, 120:1527-1533.

Campos H (1983) Estatística experimental não-paramétrica. $4^{\text {a }}$ ed. Piracicaba, Esalq. 349p.

Cargnelutti filho A, Lúcio AD \& Lopes SJ (2009) Experimentação agrícola e florestal. Santa Maria, UFSM. 204p.

Chui QS, Bispo JMA \& Iamashita CO (2004) O papel dos programas interlaboratoriais para a qualidade dos resultados analíticos. Química Nova, 27:993-1003.

CQFS - Comissão de Química e Fertilidade do Solo (2004) Manual de recomendação de adubação e de calagem para os Estados do Rio Grande do Sul e Santa Catarina. 10 $0^{\mathrm{a}}$ ed. Porto Alegre, SBCS/ NRS. 400p.

Crespo AA (2009) Estatística Fácil. 19ª ed. São Paulo, Editora Saraiva. 218p.

IAC (2006) Ensaio de proficiência IAC para laboratórios de análise de solo para fins agrícolas. Disponível em: <http:// lab.iac.sp.gov.br/normas6.pdf>. Acessado em: 16 de dezembro de 2011.

INMETRO (2007) Vocabulário internacional de termos de metrologia legal. $5^{\mathrm{a}}$ ed. Rio de Janeiro, SENAI. 72p.

Quaggio JA (1987) Programa de laboratórios de análise de solo do Estado de São Paulo. Campinas, SBCS. 9p. (Boletim Técnico, 12).

Quaggio JA, Cantarella H \& Raij van B (1994) Evolution of the analytical quality of soil testing laboratories integrated in a sample exchange program. Communications in Soil Sciences and Plant Analysis, 25:1007-1014.

Schlindwein JA \& Gianello C (2008) Calibração de métodos de determinação de fósforo em solos cultivados sob sistema plantio direto. Revista Brasileira de Ciência do Solo, 32:2037-2049.

Wiethölter S (2008) Os 40 anos da ROLAS e sua contribuição para a Ciência do Solo no Rio Grande do Sul e em Santa Catarina. In: Reunião Sul-brasileira de Ciência do Solo, Santa Maria. Anais, SBCS/NRS. CD-ROM. 